\title{
Bulgarie
}

\section{Yaroslava Genova}

\section{(2) OpenEdition}

Journals

Édition électronique

URL : https://journals.openedition.org/rdctss/1256

DOI : $10.4000 /$ rdctss. 1256

ISSN : 2262-9815

\section{Éditeur}

Centre de droit comparé du travail et de la sécurité sociale

\section{Édition imprimée}

Date de publication : 1 avril 2020

Pagination : 174-175

ISSN : 2117-4350

\section{Référence électronique}

Yaroslava Genova, «Bulgarie », Revue de droit comparé du travail et de la sécurité sociale [En ligne], 1 |

2020, mis en ligne le 01 novembre 2021, consulté le 11 novembre 2021. URL : http://

journals.openedition.org/rdctss/1256 ; DOI : https://doi.org/10.4000/rdctss.1256

\section{(c) $(1)(9)$}

Revue de droit comparé du travail et de la sécurité sociale est mise à disposition selon les termes de la Licence Creative Commons Attribution - Pas d'Utilisation Commerciale - Pas de Modification 4.0 International. 


\section{YAROSLAVA GENOVA}

UNIVERSITÉ DE PLOVDIV «PAISSII HILENDARSKI »

\section{I - LE CODE DE LA SÉCURITÉ SOCIALE}

Durant le second semestre 2019, les modifications les plus substantielles du Code de la Sécurité sociale sont le fait de la Loi budgétaire de Sécurité sociale pour 2020, qui fixe la pension de vieillesse minimale à 219,43 Leva (environ 112 euros) et prévoit son augmentation à 250 Leva (environ 128 euros) dès le 1 er juillet 2020. Cette loi précise également les obligations des assureurs concernant les documents du dossier des salariés assurés. Comme ces documents peuvent servir de preuves des droits des assurés, qui reposent sur des périodes de cotisations, les assureurs sont obligés de les conserver durant 50 ans. Est également précisée l'obligation de l'assureur qui cesse définitivement son activité de déposer les documents du dossier des salariés auprès d'une branche territoriale de l'Institut National des Assurances.

Parmi les autres amendements qui modifient certains articles du Code, on peut aussi mentionner quelques lois complétant les articles $343 \mathrm{c}$ et $343 \mathrm{~g}$, relatifs aux assurances des personnes ayant cessé leur travail pour une institution ou un organe de l'Union Européenne et qui sont donc sorties des systèmes de pensions des fonctionnaires de I'UE. Si ces personnes sont nées après le 31 décembre 1959, elles disposent normalement d'un triple choix pour transférer leurs droits acquis dans les schémas de pensions des fonctionnaires de I'UE.

Toutefois, cette règle vient d'être complétée pour fonctionner en cohérence avec la possibilité existant déjà depuis quelques années pour les personnes assurées en Bulgarie et nées après le 31 décembre 1959. En effet, même si le deuxième pilier des retraites de vieillesse continue de porter le nom d'« obligatoire ", il est déjà possible d'y renoncer et de transférer la cotisation de $5 \%$ - normalement versée vers un Fonds Universel de pensions complémentaires obligatoires - vers le Fonds «Pensions » de I'Institut National des Assurances. A cet égard, le législateur vient de préciser que les ex-fonctionnaires des institutions européennes nés après le 31 décembre 1959, qui redeviennent des personnes assurées en Bulgarie et qui ont effectué le choix de renoncer au second pilier des retraites de vieillesse, peuvent alors uniquement transférer leurs droits acquis des schémas de pensions des fonctionnaires de I'UE vers le Fonds "Pensions » de l'Institut National des Assurances. Les deux autres possibilités de transfert restent évidemment les mêmes.

\section{II - LA LOI SUR L'AIDE PERSONNELLE}

L'année sociale 2019 fut placée sous le signe d'un nouveau " paquet législatif " concernant les personnes vulnérables du point de vue social. La Loi des services sociaux devait initialement entrer en vigueur le $1^{\text {er }}$ janvier 2020, mais l'activité de quelques organisations non gouvernementales, en particulier de parents d'enfants handicapés, ont exercé une influence considérable sur l'opinion publique en défaveur de ce texte. Des doutes et des craintes furent ainsi répandus, propageant l'idée selon laquelle certains services sociaux et mesures destinés aux mineurs portaient atteinte aux droits des parents. Le ministère du travail et de la politique sociale a tenté de prouver l'absurdité de ces informations. Mais ces tensions, concomitantes à des difficultés de la Sécurité sociale, ont conduit le Ministre du travail à donner sa démission début décembre 2019. 
La nouvelle Ministre, Mme Satcheva, a entrepris de renouer le dialogue avec les organisations de parents mécontentes, ce qui a permis d'aboutir à la décision de reprogrammer l'entrée en vigueur de la loi. Le Parlement a ainsi voté la nouvelle date d'entrée en vigueur de la Loi des services sociaux au $1^{\text {er }}$ juillet 2020. Il est néanmoins probable qu'avant cette date soient effectuées quelques modifications dans la loi même, pour mieux prendre en compte les intérêts des familles. Par ailleurs, en 2019, la loi sur l'aide personnelle est entrée en vigueur. Elle réglemente la possibilité des personnes handicapées de se faire assister par une tierce personne durant un certain nombre d'heures par mois, selon les degrés de la perte d'autonomie et selon leurs besoins concrets, tels que définis grâce une évaluation personnalisée.

Cette nouvelle loi s'adresse aux personnes handicapées sans exclure quiconque en raison du degré de l'incapacité de travail, de l'âge ou du revenu. Sa logique est plutôt hybride. Elle repose, d'une part sur les droits des personnes handicapées, l'égalité des chances, la dignité de la personne humaine et l'intégration sociale - autant de principes consacrés par la Constitution bulgare et divers actes internationaux - et, d'autre part, sur la logique de solidarité nationale d'inspiration Beveridgienne ${ }^{1}$ face aux coûts des différents soins nécessaires pour assurer une vie décente et une autonomie maximale aux personnes handicapées.

Peut être bénéficiaire de l'aide personnelle toute personne handicapée dont le handicap a réduit sa capacité à se procurer elle-même les soins au quotidien et a, de ce fait, entraîné une dépendance à l'aide d'une personne tierce pour compenser son déficit fonctionnel, soutenir l'exercice de ses droits fondamentaux et lui permettre de participer pleinement à la vie sociale.

La personne handicapée bénéficie de cette aide en nature (profitant directement des services et des soins procurés par l'assistant choisi). Le coût de l'aide personnelle, y compris la rémunération des heures de travail effectuées par chaque assistant, est financé par le Budget de l'État et par d'autres sources complémentaires, tels les Fonds structurels et d'investissement de I'UE. Les personnes handicapées à $90 \%$ ou plus qui reçoivent une pension de la Sécurité sociale et un complément d'aide à une tierce personne, y compris lorsqu'elles ont un âge avancé, peuvent choisir de profiter de l'aide individualisée d'un assistant dans le cadre de la nouvelle Loi d'assistance personnelle. Dans ce cas, elles sont obligées de le déclarer auprès de l'Institut National des Assurances.

Ainsi, leur complément de pension pour l'aide d'une tierce personne sera directement versé à l'Agence d'aide sociale en charge du contrat de travail de l'assistant choisi, ce qui confirme l'inspiration plutôt Beveridgienne de la nouvelle aide personnelle. De même, s'il s'agit d'un enfant handicapé, son représentant légal doit consentir le transfert direct à la mairie où l'enfant réside d'une partie (pas plus de 380 Leva, soit $195 €$ ) de l'aide spéciale pour enfants handicapés reçue par le mineur, afin de contribuer au financement de l'aide personnelle. L'octroi et le financement de cette nouvelle aide individuelle ont débuté le $1^{\text {er }}$ septembre 2019. L'efficacité de cette nouvelle forme d'aide pour les personnes handicapées reste à déterminer, tout comme la satisfaction des bénéficiaires, dont une majorité a réellement combattu en faveur de davantage de possibilités d'autonomie pour les personnes handicapées et leurs familles.

1 William Beveridge, économiste et homme politique britannique (1879-1963). 